\title{
$\mathrm{SiH}_{4}$ 与 $\mathrm{AB}$ 型卤素互化物之间的反转氢键
}

\author{
刘红*陈燕芹杨玉琼 \\ (毕节学院化学系, 贵州 毕节 551700)
}

\begin{abstract}
摘要：采用从头算方法对 $\mathrm{SiH}_{4}$ 与 $\mathrm{AB}$ 型卤素互化物 $(\mathrm{ClF} 、 \mathrm{BrF} 、 \mathrm{IF} 、 \mathrm{ICl} 、 \mathrm{IBr} 、 \mathrm{BrCl})$ 形成的复合物的结构特征及本 质进行了探讨. 在 MP2/6-311++G(3d,3p)水平上优化复合物的分子结构, 并进行频率验证. 通过分子间距离、自然 键轨道 $(\mathrm{NBO})$ 净电荷迁移数及分子图, 确认 $\mathrm{SiH}_{4}$ 与卤素互化物形成反转氢键型复合物. 在 $\mathrm{MP} 2 / 6-311++\mathrm{G}(3 d, 3 p)$ 水平上进行基组重叠误差(BSSE)校正后的作用能为 $-5.113--9.468 \mathrm{~kJ} \cdot \mathrm{mol}^{-1}$. 用对称匹配微扰理论(SAPT)对作 用能进行分解, 结果显示, 诱导能对总吸引能的贡献在 $55.0 \%$ 到 $72.2 \%$ 之间, 是最主要的贡献部分, 静电能和色散 能对总吸引能的贡献都小于 $25.0 \%$.
\end{abstract}

关键词： 反转氢键；对称匹配微扰理论； $\mathrm{SiH}_{4} ;$ 卤素互化物 中图分类号：0641

\section{Inverse Hydrogen Bond between Silicane and AB-Type Interhalogen Compounds}

\author{
LIU Hong * CHEN Yan-Qin YANG Yu-Qiong \\ (Department of Chemistry, Bijie University, Bijie 551700, Guizhou Province, P. R. China)
}

\begin{abstract}
Intermolecular complexes of silicane with $\mathrm{AB}$-type interhalogen compounds $(\mathrm{ClF}, \mathrm{BrF}, \mathrm{IF}, \mathrm{ICl}, \mathrm{IBr}, \mathrm{BrCl})$ were examined using $a b$ initio calculations performed at the second-order Møller-Plesset perturbation approximation with the $6-311++\mathrm{G}(3 d, 3 p)$ basis set. Frequency calculations were also run using the same level of theory. Inverse hydrogen bonds were formed in the complexes of silicane with AB-type interhalogen compounds as determined by the geometrical criteria and the natural bond orbital (NBO) net charge transfer number as well as the molecular graphs. The calculated binding energies of the complexes using MP2/6-311++ $\mathrm{G}(3 d, 3 p)$ methods and corrected by the basis-set superposition error (BSSE) were -5.113 to $-9.468 \mathrm{~kJ} \cdot \mathrm{mol}^{-1}$. Decomposition of the interaction energies was carried out using symmetry adapted perturbation theory (SAPT). The results indicate that the contribution of the induction energy to the total attractive energy ranges from $55.0 \%$ to $72.2 \%$, which are the primary contribution to the total attractive energy. The contribution of electrostatic energy and dispersion energy to the total attractive energy are less than $25.0 \%$.
\end{abstract}

Key Words : Inverse hydrogen bond; Symmetry adapted perturbation theory; Silicane;

Interhalogen compound

氢键是很多化学和生物体系中的重要现象, 在 有十分重要的意义 ${ }^{[5-6]}$. 常规的氢键 $X-\mathrm{H}^{*} \cdots \mathrm{Y}$ 是在 分子识别、超分子自组装等方面都有重要的应用 ${ }^{[1-4]}$. 一个质子给体(例如一个 $\mathrm{O}-\mathrm{H}$ 或 $\mathrm{N}-\mathrm{H}$ 基团)和一 由于分子间相互作用在生命科学和材料科学中的关 个质子受体原子(例如带有孤对电子的 $\mathrm{O}$ 或 $\mathrm{N}$ 原 键作用, 因此对其作用机制、作用本质的理论研究具 子)之间形成, $\mathrm{X}$ 和 $\mathrm{Y}$ 都是 $\mathrm{F} 、 \mathrm{O} 、 \mathrm{~N} 、 \mathrm{Cl}$ 等电负性较高

Received: January 25, 2010; Revised: April 21, 2010; Published on Web: June 11, 2010.

${ }^{*}$ Corresponding author. Email: hliu315@126.com; Tel: +86-857-8331343.

The project was supported by the Natural Science Foudation of Guizhou Education Department, China (2008075), and Science Foundation of Bijie

University, China (20082006).

贵州省教育厅自然科学研究基金(2008075)及毕节学院科学研究基金(20082006)资助项目

C. Editorial office of Acta Physico-Chimica Sinica 


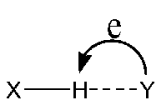

a

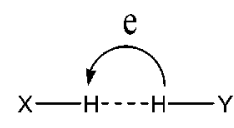

b

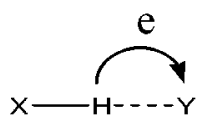

c
图 1 几种氢键示意图

Fig.1 Schematic diagram of several hydrogen bonds

的原子. 在常规氢键体系中, 氢原子是电子受体(如 图 1a). 近年来发现的二氢键 $(\mathrm{X}-\mathrm{H} \cdots \mathrm{H}-\mathrm{Y})^{\text {中 }}{ }^{[7-13]}$, 一 个氢原子是电子给体, 另一个是电子受体 (见图 $1 b$ ). 还有一种氢键, 氢原子是电子给体, 其它的非氢原子 作为电子受体(见图 1c), Rozas ${ }^{[14]}, \mathrm{Li}^{[15]}$ 和 Blanco ${ }^{[16]}$ 等 把这样的体系称之为反转氢键 (inverse hydrogen bond), 并对理论模型 $\mathrm{Li}-\mathrm{H} \cdots \mathrm{Li}-\mathrm{H}, \mathrm{H}-\mathrm{Be}-\mathrm{H} \cdots$ $\mathrm{Li}-\mathrm{H}, \mathrm{H}-\mathrm{Be}-\mathrm{H} \cdots \mathrm{Li}-\mathrm{CH}_{3}, \mathrm{HBH}_{3} \cdots \mathrm{Li}-\mathrm{H}$ 进行探 讨. 据文献检索, 对反转氢键体系相互作用的本质还 不清楚.

$\mathrm{SiH}_{4}$ 作为一种原料气体被广泛地应用于化学 气象沉积法中, 在基质隔离技术中, 它也被作为一种 基质气体. 认识 $\mathrm{SiH}_{4}$ 形成复合物的本质和规律, 有 助于超分子的研究, 并且为它们更好的利用打下基 础 ${ }^{[17-18]}$. 本文系统研究了 $\mathrm{SiH}_{4}$ 与 $\mathrm{AB}$ 型卤素互化物 $(\mathrm{ClF} 、 \mathrm{BrF} 、 \mathrm{IF} 、 \mathrm{ICl} 、 \mathrm{IBr} 、 \mathrm{BrCl})$ 形成的复合物的结构特 征、能量变化规律、能量变化与 $\mathrm{Si}-\mathrm{H}$ 的伸缩振动 的关系, 并使用 SAPT 法探讨静电能、诱导能、色散 能对该类复合物作用能的贡献, 以期揭示该类复合 物的作用本质.

\section{1 计算方法}

所有单体和复合物优化的几何结构都是在 $\mathrm{MP} 2 / 6-311++\mathrm{G}(3 d, 3 p)$ 水平上优化, 然后在同样的计 算水平计算频率, 确定得到的是稳定结构. 作用能、 BSSE 和 BSSE 校正后的作用能也在 MP2/6-311++ $\mathrm{G}(3 d, 3 p)$ 水平上计算得到. 拓扑指标采用 Gaussian 98 程序 ${ }^{[19]}$ 中的 AIM 模块完成. 采用 MP2/6-311++ $\mathrm{G}(3 d, 3 p)$ 水平得到的波函数, 用 AIM2000 软件 ${ }^{[20]}$ 作 出分子图.

SAPT 是一种研究分子间相互作用本质的重要 方法. SAPT 将相互作用能分解为静电能、诱导能、色 散能和交换能之和. SAPT有不同的计算公式, 常用 的有 SAPT2、SAPT和SAPT0. 由于SAPT2 的相互作 用能近似等于二级微扰方法 (MP2), 因此本文中 能量分解分析采用 SAPT2002 程序 ${ }^{[21]}$, 在 SAPT2级 别, 用同样的基函数对 $\mathrm{SiH}_{4}$ 与 $\mathrm{AB}$ 型卤素互化物
$(\mathrm{ClF} 、 \mathrm{BrF} 、 \mathrm{IF} 、 \mathrm{ICl} 、 \mathrm{IBr} 、 \mathrm{BrCl})$ 进行能量分解分析.

\section{2 结果与讨论}

\section{1 几何构型、作用能及拓扑性质}

对 $\mathrm{SiH}_{4}$ 与 $\mathrm{AB}$ 型卤素互化物 $(\mathrm{ClF} 、 \mathrm{BrF} 、 \mathrm{IF} 、 \mathrm{ICl}$ 、 $\mathrm{IBr} 、 \mathrm{BrCl})$ 形成的复合物的不同的初始构型在 MP2/ $6-311++\mathrm{G}(3 d, 3 p)$ 水平上进行几何优化和频率验证, 最后得到没有虚频的结构, 如图 2 所示. 结构参数列 于表 1 中. 从图 2 可以看到, $\mathrm{AB}$ 型卤素互化物与 $\mathrm{SiH}_{4}$ 形成复合物时, 只与其中的一个氢作用.

表 1 为在 MP2/6-311++g $(3 d, 3 p)$ 水平上优化并 经过频率验证得到的硅烷与卤素互化物作用的稳定 结构的几何参数. 从表 1 可以看出, 形成复合物后, 与卤素互化物直接作用的 $\mathrm{Si}-\mathrm{H}(1)$ 键都变长, 键长 变化最大的是 $\mathrm{SiH}_{4} \cdots \mathrm{IF}$ 体系, $\mathrm{Si}-\mathrm{H}(1)$ 键键长增加 了 $0.0016 \mathrm{~nm}$. 卤素互化物的键长也发生了较大的 变化, 变化最大的为 $\mathrm{BrF}$, 形成复合物后, 键长增加 了 $0.0013 \mathrm{~nm}$, 其余体系中变化最小的也达 0.0008 $n$ m. $H(1) \cdots X(1)$ 间的距离都大于 $H$ 原子和 $X$ 原子共 价半径之和而小于它们的范德华半径之和 $(\mathrm{H}-\mathrm{Cl}$, $\mathrm{H}-\mathrm{Br}, \mathrm{H}-\mathrm{I}$ 的共价半径之和分别为 0.1274 、 $0.1408 、 0.1609 \mathrm{~nm}$; 范德华半径之和分别为 0.295 、

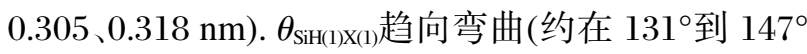
之间), $\theta_{\mathrm{H}(1) \mathrm{X}(1) \mathrm{X}(2)}$ 趋向直线(约在 $177^{\circ}$ 到 $180^{\circ}$ 之间).

氢键的形成常根据几何参数来判断. 简单地说, 质子给体和受体原子间的距离应大于共价半径之和 而小于范德华半径之和; 形成复合物后, 质子给体 的键长应该增加. 根据以上规则可以认为硅烷与卤 素互化物间都形成了氢键.

在 $\mathrm{MP} 2 / 6-311++\mathrm{G}(3 d, 3 p)$ 水平上计算得到的 $\mathrm{SiH}_{4}$ 与 $\mathrm{AB}$ 型卤素互化物形成的复合物相互作用能 $(\Delta E)$, 基组重叠误差(BSSE), 基组重叠误差校正的相 互作用能 $\left(\Delta E_{\mathrm{cp}}\right)$ 列在表 2 中. 从表 2 可以得出: $\mathrm{SiH}_{4} \cdots$

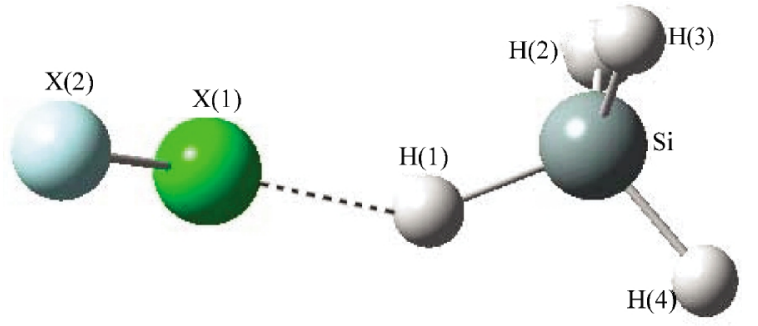

图 $2 \mathrm{SiH}_{4}$ 与 $\mathrm{AB}$ 型卤素互化物形成的复合物的 优化几何构型

Fig.2 Optimized geometries for complexes of $\mathrm{SiH}_{4}$ with AB-type interhalogen compounds 
表 1 优化得到的 $\mathrm{SiH}_{4}$ 与 $\mathrm{AB}$ 型卤素互化物形成的复合物的几何参数

Table 1 Optimized geometric parameters for complexes of $\mathrm{SiH}_{4}$ with $\mathrm{AB}$-type interhalogen compounds

\begin{tabular}{|c|c|c|c|c|c|c|}
\hline & $\mathrm{SiH}_{4} \cdots \mathrm{ClF}$ & $\mathrm{SiH}_{4} \cdots \mathrm{BrF}$ & $\mathrm{SiH}_{4} \cdots \mathrm{IF}$ & $\mathrm{SiH}_{4} \cdots \mathrm{ICl}$ & $\mathrm{SiH}_{4} \cdots \mathrm{IBr}$ & $\mathrm{SiH}_{4} \cdots \mathrm{BrCl}$ \\
\hline$R_{\mathrm{H}(1) \cdots \mathrm{X}(1)} / \mathrm{nm}$ & 0.2340 & 0.2282 & 0.2412 & 0.2605 & 0.2716 & 0.2562 \\
\hline$R_{\mathrm{Si}-\mathrm{H}(1)} / \mathrm{nm}$ & 0.1482 & 0.1488 & 0.1489 & 0.1482 & 0.1481 & 0.1480 \\
\hline$\Delta R_{\mathrm{Si}-\mathrm{H}(\mathrm{I})} / \mathrm{nm}$ & 0.0009 & 0.0015 & 0.0016 & 0.0009 & 0.0008 & 0.0007 \\
\hline$R_{\mathrm{X}(1)-\mathrm{X}(2)} / \mathrm{nm}$ & 0.1668 & 0.1801 & 0.1947 & 0.2372 & 0.2515 & 0.1176 \\
\hline$\Delta R_{\mathrm{X}(1)}-\mathrm{X}(2) / \mathrm{nm}$ & 0.0010 & 0.0013 & 0.0011 & 0.0011 & 0.0008 & 0.0009 \\
\hline$\theta_{\mathrm{SiH}(1) \mathrm{X}(1)} /\left(^{\circ}\right)$ & 131.9 & 134.3 & 138.9 & 146.7 & 136.9 & 134.6 \\
\hline$\theta_{\mathrm{H}(1) \mathrm{X}(1) \mathrm{X}(2)} /\left(^{\circ}\right)$ & 177.7 & 177.0 & 176.9 & 178.0 & 179.0 & 178.5 \\
\hline
\end{tabular}

calculated at the MP2/6-311++G(3d,3p) level; $\Delta R=R($ in complex $)-R($ in isolated molecule $)$; in isolated $\mathrm{SiH}_{4}, R_{\mathrm{Si}-\mathrm{H}}=0.1473 \mathrm{~nm}$

$\mathrm{IF}$ 复合物的作用能最大, 作用能 $\Delta E_{\mathrm{cp}}$ 为 $-9.468 \mathrm{~kJ}$. $\mathrm{mol}^{-1} ; \Delta E_{\mathrm{cp}}$ 最小的是 $\mathrm{SiH}_{4} \cdots \mathrm{IBr}$ 复合物, 基组重叠误 差校正后的作用能是 $-5.113 \mathrm{~kJ} \cdot \mathrm{mol}^{-1}$. 表 2 中也列出 了 $\mathrm{Si}-\mathrm{H}(1)$ 键的伸缩振动频率, 在所有的复合物中 $\mathrm{Si}-\mathrm{H}(1)$ 键的伸缩振动频率都发生了红移, 其中 $\mathrm{SiH}_{4} \cdots \mathrm{IF}$ 复合物的红移程度最大, 达到 $113.79 \mathrm{~cm}^{-1}$, $\mathrm{SiH}_{4} \cdots \mathrm{BrCl}$ 复合物的红移程度最小, 为 $44.87 \mathrm{~cm}^{-1}$, $\mathrm{Si}-\mathrm{H}$ 键的红移程度与作用能的变化趋势基本一致.

除了根据几何参数判断是否形成反转氢键外, 我们也采用键鞍点的拓扑参数判断 $\mathrm{SiH}_{4}$ 与 $\mathrm{AB}$ 型 卤素互化物形成复合物的成键情况. 表 3 中列出了 $\mathrm{H}(1) \cdots \mathrm{X}(1), \mathrm{X}(1)-\mathrm{X}(2)$ 及 $\mathrm{Si}-\mathrm{H}(1)$ 键的键临界点的 电子密度 $\left(\rho_{c}\right)$ 和电子密度的拉普拉斯量 $\left(\nabla^{2} \rho_{c}\right)$, 也列 出了单体中 $\mathrm{X}(1)-\mathrm{X}(2), \mathrm{Si}-\mathrm{H}(1)$ 键的键临界点的 $\rho_{\mathrm{c}}$ 和 $\nabla^{2} \rho_{\mathrm{c}}$ 值. 根据 Bader ${ }^{[22]}$ 的分子中原子(AIM)理论, 形成氢键的条件是键临界点的 $\rho_{\mathrm{c}}$ 在 0.002-0.04 a.u. 之间, 相应的 $\nabla^{2} \rho_{\mathrm{c}}$ 在 0.024-0.139 a.u.之间, 键临界 点的 $\rho_{\mathrm{c}}$ 与原子之间的成键强度有关, $\rho_{\mathrm{c}}$ 值越大, 说明

\section{表 2 复合物的相互作用能 $(\Delta E)$ 、基组重叠误差 $(\mathrm{BSSE})$ 、 基组重叠误差校正后的相互作用能 $\left(\Delta \boldsymbol{E}_{\mathrm{cp}}\right)$ 及复合物中 $\mathrm{Si}-\mathrm{H}(1)$ 的伸缩振动频率 $(\nu)$}

Table 2 Interaction energies $(\Delta E)$, BSSE, interaction energy corrected with BSSE ( $\left.\Delta E_{\mathrm{cp}}\right)$ of the complexes and stretching vibrational frequencies $(\nu)$ of the $\mathrm{Si}-\mathbf{H}(\mathbf{1})$ subunits

\begin{tabular}{|c|c|c|c|c|c|}
\hline \multirow{2}{*}{ Complex } & \multirow{2}{*}{$\frac{\Delta E}{\left(\mathrm{~kJ} \cdot \mathrm{mol}^{-1}\right)}$} & \multirow{2}{*}{$\frac{\mathrm{BSSE}}{\left(\mathrm{kJ} \cdot \mathrm{mol}^{-1}\right)}$} & \multirow{2}{*}{$\frac{\Delta E_{\mathrm{cp}}}{\left(\mathrm{kJ} \cdot \mathrm{mol}^{-1}\right)}$} & \multicolumn{2}{|c|}{$\mathrm{Si}-\mathrm{H}(1)$} \\
\hline & & & & $\nu_{\text {comp }} / \mathrm{cm}^{-1}$ & $\Delta \nu / \mathrm{cm}^{-1}$ \\
\hline $1 x_{4}$ & -9.782 & 2 & -5.870 & 2244.16 & 6 \\
\hline $\mathrm{iH}_{4} \cdots \mathrm{BrF}$ & -13.029 & 3.745 & -9.284 & 219691 & 110.95 \\
\hline $\mathrm{iH}_{4} \cdots \mathrm{IF}$ & -16.062 & 6.594 & -9.468 & 2194.06 & 113.79 \\
\hline $\mathrm{iH}_{4} \cdots \mathrm{ICl}$ & -11.355 & 5.611 & -5.745 & 2247.69 & 00.1 \\
\hline $\mathrm{SiH}_{4} \cdots \mathrm{IBr}$ & -9.577 & 4.464 & -5.113 & 2251.22 & 56.64 \\
\hline $\mathrm{SiH}_{4} \cdots \mathrm{BrCl}$ & -8.975 & 3.841 & -5.134 & 2262.99 & 44.87 \\
\hline
\end{tabular}

calculated at the MP2/6-311++G(3d,3p) level; $\Delta \nu=\nu_{\text {free }}-\nu_{\text {comp }}$, $\nu_{\text {free }}=2307.86 \mathrm{~cm}^{-1}$
该化学键的强度越大; 反之, 则越小. 从表 3 中可以 看出, 本文所探讨的体系中 $\rho_{\mathrm{c}}$ 从 0.013 到 0.022 a.u., $\nabla{ }^{2} \rho_{\mathrm{c}}$ 从 0.034 到 0.068 a.u., 这说明所研究的 $\mathrm{SiH}_{4}$ 与 $\mathrm{AB}$ 型卤素互化物形成的复合物间的反转氢键在强 度上相当于常规氢键. 对 $\mathrm{SiH}_{4} \cdots \mathrm{I}-\mathrm{Y}(\mathrm{Y}=\mathrm{F} 、 \mathrm{Cl} 、 \mathrm{Br})$ 体 系, 电荷密度增大的趋势与作用能增大的趋势一致, 即 $\rho_{\mathrm{c}}$ 越大, 作用能越大.

图 3 是 $\mathrm{SiH}_{4}$ 与 $\mathrm{AB}$ 型卤素互化物形成的复合 物的 AIM 分子图, 根据 Bader ${ }^{[22]}$ 提出的 AIM 理论, 如果相邻的两个原子之间存在着成键作用, 那么一 定有一条从键鞍点出发连接两原子的键径存在. 从 图 3 可以看出,卤素原子和 $\mathrm{SiH}_{4}$ 的一个氢原子之间 存在一个键鞍点且由两条键径分别与卤素原子和氢 原子相连接, 说明形成了反转型氢键.

\section{2 自然键轨道 NBO 分析}

氢键的形成伴随有微小的电子移动. 最近几年,

表 3 复合物的拓扑参数

Table 3 Topological parameters of the complexes

\begin{tabular}{lcccccccc}
\hline \multirow{2}{*}{ Complex } & \multicolumn{2}{c}{$\mathrm{Si}-\mathrm{H}(1)$} & & \multicolumn{2}{c}{$\mathrm{H}(1) \cdots \mathrm{X}(1)$} & & \multicolumn{2}{c}{$\mathrm{X}(1)-\mathrm{X}(2)$} \\
\cline { 2 - 3 } \cline { 7 - 8 } & $\rho_{\mathrm{c}}$ & $\nabla^{2} \rho_{\mathrm{c}}$ & & $\rho_{\mathrm{c}}$ & $\nabla^{2} \rho_{\mathrm{c}}$ & & $\rho_{\mathrm{c}}$ & $\nabla^{2} \rho_{\mathrm{c}}$ \\
\hline $\mathrm{SiH}_{4} \cdots \mathrm{ClF}$ & 0.117 & 0.231 & & 0.017 & 0.057 & & 0.188 & -0.052 \\
& $(0.122)$ & $(0.229)$ & & & & $(0.187)$ & $(0.043)$ \\
$\mathrm{SiH}_{4} \cdots \mathrm{BrF}$ & 0.114 & 0.229 & & 0.022 & 0.068 & & 0.144 & 0.261 \\
& $(0.122)$ & $(0.229)$ & & & & $(0.147)$ & $(0.256)$ \\
$\mathrm{SiH}_{4} \cdots \mathrm{IF}$ & 0.113 & 0.224 & 0.022 & 0.055 & & 0.116 & 0.490 \\
& $(0.122)$ & $(0.229)$ & & & & $0.119)$ & $(0.449)$ \\
$\mathrm{SiH}_{4} \cdots \mathrm{ICl}$ & 0.117 & 0.231 & 0.015 & 0.039 & & 0.091 & 0.058 \\
& $(0.122)$ & $(0.229)$ & & & & $0.090)$ & $(0.056)$ \\
$\mathrm{SiH}_{4} \cdots \mathrm{IBr}$ & 0.118 & 0.231 & 0.013 & 0.034 & & 0.085 & -0.008 \\
& $(0.122)$ & $(0.229)$ & & & & $0.082)$ & $(0.009)$ \\
$\mathrm{SiH}_{4} \cdots \mathrm{BrCl}$ & 0.118 & 0.231 & 0.013 & 0.041 & & 0.122 & -0.037 \\
& $(0.122)$ & $(0.229)$ & & & & $(0.117)$ & $(0.011)$ \\
\hline
\end{tabular}

calculated at the MP2/6-311++G(3d,3p) level; $\rho_{\mathrm{c}}$ : electron density at bond critical point (BCP), $\nabla^{2} \rho_{c}$ : electron density Laplacians, $\rho_{\mathrm{c}}$ and $\nabla^{2} \rho_{\mathrm{c}}$ in a.u.; The values corresponding for monomers are included in parentheses. 

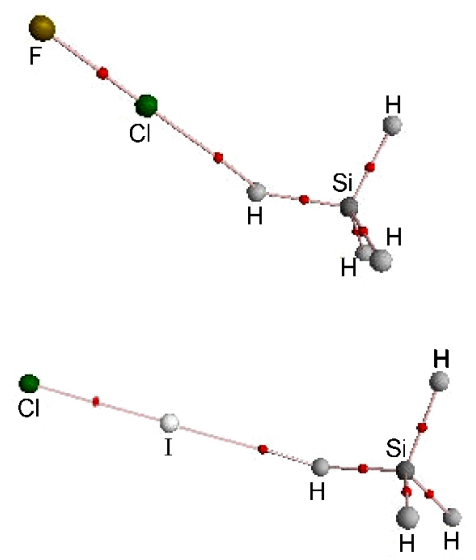
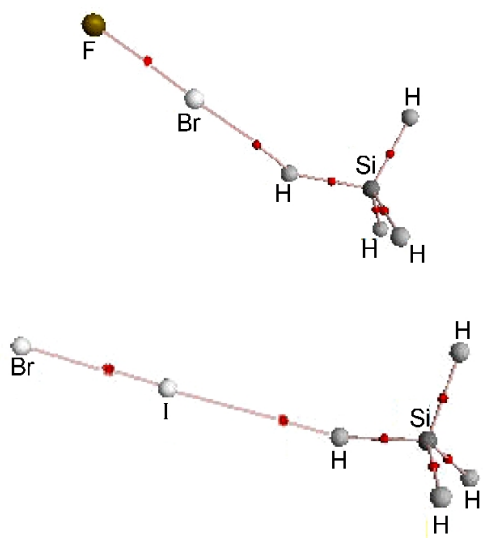
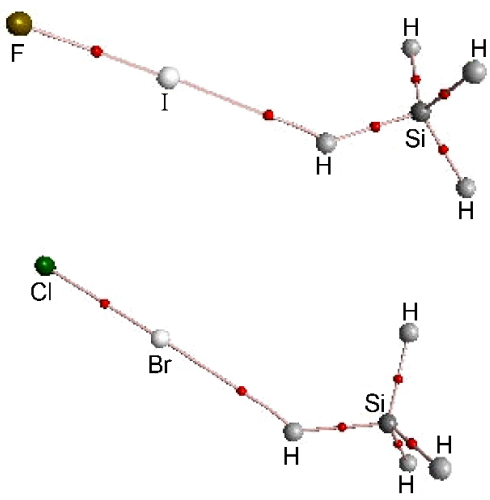

图 $3 \mathrm{SiH}_{4}$ 与 $\mathrm{AB}$ 型卤素互化物形成的复合物的分子图

Fig.3 Molecular graphs for complexes of $\mathrm{SiH}_{4}$ with AB-type interhalogen compounds

广泛使用 NBO 方法分析原子电荷 ${ }^{[23-24]}$; 这个方法计 算结果不受基组和弥散函数的影响, 研究结果比较 可靠. 所以, 本论文采用 NBO 方法分析复合物单体 之间电荷的迁移. 表 4 是在 MP2/6-311++G $(3 d, 3 p)$ 水平上计算的 $\mathrm{NBO}$ 净电荷迁移数, 从表中可以看 出, 所有的复合物中 $\mathrm{SiH}_{4}$ 都是作为电子给予体, 电 子迁移的方向与常规氢键相反, 符合 Rozas 等 ${ }^{[14]}$ 对 反转氢键的定义. 对我们所研究的体系, 净电荷迁移 数从 0.0080 到 0.0246 a.u., 最大的是 $\mathrm{SiH}_{4} \cdots \mathrm{IF}$ 体系, 为 0.0246 a.u..

\section{3 能量分解分析}

为了研究 $\mathrm{SiH}_{4}$ 与 $\mathrm{AB}$ 型卤素互化物形成的复 合物相互作用的本质, 及各种能量对作用能的贡献 变化规律, 我们进行了能量分解分析. $\mathrm{SiH}_{4}$ 与 $\mathrm{AB}$ 型 卤素互化物形成的复合物采用 MP2/6-311++G(3d, $3 p$ )水平上优化得到的稳定结构, 用 SAPT2002 程序 在 SAPT2 级别分解其作用能, 结果见表 5. 为了便 于与 MP2 计算得到的结果比较, 所用的基组均为 6$311++\mathrm{G}(3 d, 3 p)$. 受计算条件的限制, $\mathrm{SiH}_{4} \cdots \mathrm{IBr}$ 体系 的能量分解没有完成.

表 5 中 $E_{\mathrm{int}, \mathrm{r}}^{(\mathrm{corr} 2)}$ 表示电子相关能, $E_{\mathrm{int}}^{\mathrm{HF}}=E_{\mathrm{elst}}^{(\mathrm{d})}+E_{\mathrm{exch}}^{(\mathrm{d} 10)}+E_{\mathrm{ind}, \mathrm{r}}^{(20)}+$ $E_{\text {exch-ind, } \mathrm{r}}^{(20)}+\delta E_{\mathrm{int}, \mathrm{r}}^{\mathrm{HF}}$, 表示Hartree-Fock作用能, $E_{\mathrm{int}}(\mathrm{SAPT} 2)=$ $E_{\mathrm{int}}^{\mathrm{HF}}+E_{\mathrm{int}, \mathrm{r}}^{(\mathrm{corr})}$, 表示标准 SAPT 作用能. 其中 $E_{\mathrm{elst}}^{(\mathrm{il})}$ 是经典
的静电相互作用能, $E_{\mathrm{exch}}^{(10)}$ 是 SAPT 定义的一阶交换 能, $E_{\mathrm{ind}, \mathrm{r}}^{(20)}$ 为诱导能, $E_{\mathrm{exch-nd, \textrm {r }}}^{(20)}$ 为交换诱导部分, $\delta E_{\mathrm{in}, \mathrm{r}}^{\mathrm{HF}}$ 为 所有三级和高级诱导、交换诱导的总和. 下标 “ $r$ ”表 示 $E_{\text {exch-ind, }}^{(20)}$ 和 $E_{\text {exch-nd, } \mathrm{r}}^{(20)}$ 由偶合 Hartree-Fock(CHF) 理论 得到.

在弱相互作用的研究中, 电子相关效应非常重 要 ${ }^{[25],}$, 其重要性可从 $E_{\mathrm{in,} \mathrm{r}}^{(\mathrm{corr})}$ 的数值体现出来, 从表 5 中可以看到, 反转氢键体系的电子相关能的数值都 很大, 如果从总的 SAPT2 相互作用能 $E_{\text {int }}(\mathrm{SAPT} 2)$ 中 扣除 $E_{\mathrm{inn}, \mathrm{r}}^{(\mathrm{cor} 2)}$, Hartree-Fock 级别下的相互作用能都变 成了排斥项. 因此, 包含反转氢键体系的弱相互作用 考虑电子相关是非常重要的.

为了便于说明问题, 把能量分解所得的数据归 纳成静电能 $\left(E_{\text {elst }}=E_{\text {elst }}^{(10)}+E_{\text {elst }}^{(12)}\right)$ 、交换排斥能 $\left(E_{\text {exch }}=E_{\text {exch }}^{(10)}+\right.$ $\left.E_{\text {exch-ind, } \mathrm{r}}^{(20)}+E_{\text {exch }}^{(111)}+E_{\text {exch }}^{(122)}+{ }^{t} E_{\text {exch-ind }}^{(22)}+E_{\text {exch-disp }}^{(20)}+\delta E_{\text {int, } \mathrm{r}}^{\mathrm{HF}}\right)$ 、诱导能

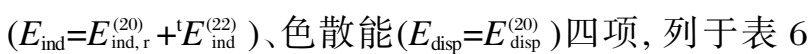
中. 从表 5 和表 6 可以得出, 在所有的体系中, 诱导 能对总吸引能(即静电、诱导和色散能之和)的贡献 都是最主要的. 在 $\mathrm{SiH}_{4} \cdots \mathrm{IF}$ 体系中, 诱导能对总吸 引能的贡献达 $72.2 \%$. 静电能、色散能对总吸引能能 的贡献都小于 $25 \%$. 交换排斥能 $\left(E_{\text {exch }}=E_{\text {exch }}^{(10)}+E_{\text {exch-nd, } \mathrm{r}}^{(20)}+\right.$ $\left.E_{\text {exch }}^{(11)}+E_{\text {exch }}^{(12)}+E_{\text {exch-ind }}^{(22)}+E_{\text {exch-disp }}^{(20)}+\delta E_{\text {int }, \mathrm{r})}^{\mathrm{HF}}\right)$ 比静电能 $\left(E_{\text {elst }}=E_{\text {elst }}^{(10)}+\right.$ $\left.E_{\text {elst }}^{(12)}\right)$ 大得多, 因此, 交换排斥能和静电吸引能的总和

表 4 复合物的 NBO 电荷迁移

Table 4 NBO charge transfer for complexes

\begin{tabular}{lcccccc}
\hline & $\mathrm{SiH}_{4} \cdots \mathrm{ClF}$ & $\mathrm{SiH}_{4} \cdots \mathrm{BrF}$ & $\mathrm{SiH}_{4} \cdots \mathrm{IF}$ & $\mathrm{SiH}_{4} \cdots \mathrm{ICl}$ & $\mathrm{SiH}_{4} \cdots \mathrm{IBr}$ & $\mathrm{SiH}{ }_{4} \cdots \mathrm{BrCl}$ \\
\hline $\mathrm{SiH}_{4}$ & +0.0119 & +0.0237 & +0.0246 & +0.0129 & +0.0091 & +0.0080 \\
$\mathrm{XY}$ & -0.0119 & -0.0237 & -0.0246 & -0.0129 & -0.0091 & -0.0080 \\
\hline
\end{tabular}


表 $5 \mathrm{SiH}_{4}$ 与 $\mathrm{AB}$ 型卤素互化物形成的复合物的 SAPT 作用能项

Table 5 SAPT interaction energy components for complexes of $\mathrm{SiH}_{4}$ with $\mathrm{AB}$-type interhalogen compounds

\begin{tabular}{|c|c|c|c|c|c|c|}
\hline & $\mathrm{SiH}_{4} \cdots \mathrm{ClF}$ & $\mathrm{SiH}_{4} \cdots \mathrm{BrF}$ & $\mathrm{SiH}_{4} \cdots \mathrm{IF}$ & $\mathrm{SiH}_{4} \cdots \mathrm{ICl}$ & $\mathrm{SiH}_{4} \cdots \mathrm{BrCl}$ & $\mathrm{HCl} \cdots \mathrm{HCl}$ \\
\hline$\overline{E_{\mathrm{int}}^{\mathrm{HF}}} /\left(\mathrm{kJ} \cdot \mathrm{mol}^{-1}\right)$ & 4.870 & 3.887 & 2.351 & 5.004 & 5.272 & -0.661 \\
\hline$E_{\mathrm{elst}}^{(10)} /\left(\mathrm{kJ} \cdot \mathrm{mol}^{-1}\right)$ & -14.435 & -24.924 & -27.472 & -15.280 & -11.196 & -10.895 \\
\hline$E_{\text {exch }}^{(10)} /\left(\mathrm{kJ} \cdot \mathrm{mol}^{-1}\right)$ & 32.430 & 53.174 & 56.877 & 33.422 & 24.790 & 15.744 \\
\hline$E_{\text {ind, } \mathrm{r}}^{(20)} /\left(\mathrm{kJ} \cdot \mathrm{mol}^{-1}\right)$ & -35.543 & -94.136 & -125.018 & -55.840 & -31.531 & -7.715 \\
\hline$E_{\text {exch-ind, } \mathrm{r}}^{(20)} /\left(\mathrm{kJ} \cdot \mathrm{mol}^{-1}\right)$ & 30.522 & 81.981 & 108.328 & 48.718 & 27.911 & 4.945 \\
\hline$\delta E_{\mathrm{int}, \mathrm{r}}^{\mathrm{HF}} /\left(\mathrm{kJ} \cdot \mathrm{mol}^{-1}\right)$ & -8.100 & -12.209 & -10.364 & -6.012 & -4.707 & -2.741 \\
\hline$E_{\mathrm{int}, \mathrm{r}}^{(\mathrm{cor} 2)} /\left(\mathrm{kJ} \cdot \mathrm{mol}^{-1}\right)$ & -10.761 & -13.845 & -14.226 & -11.728 & -10.519 & -6.042 \\
\hline$E_{\text {int }}(\mathrm{SAPT} 2) /\left(\mathrm{kJ} \cdot \mathrm{mol}^{-1}\right)$ & -5.891 & -9.958 & -11.874 & -6.720 & -5.247 & -7.017 \\
\hline$\Delta E_{\mathrm{cp}}[\mathrm{MP} 2] /\left(\mathrm{kJ} \cdot \mathrm{mol}^{-1}\right)$ & -5.870 & -9.284 & -9.468 & -5.745 & -5.134 & -6.686 \\
\hline
\end{tabular}

obtained at the MP2 level using the MP2/6-311++G(3d,3p) basis set; $E_{\mathrm{int}}^{\mathrm{HF}}$ is Hartree-Fock interaction energy, $E_{\mathrm{elst}}^{(10)}$ is the classical electrostatic energy, $E_{\text {exch }}^{(10)}$ is the first-order exchange energy, $E_{\text {ind, } \mathrm{r}}^{(20)}$ is the second-order induction energy, $E_{\text {exch-ind, } \mathrm{r}}^{(20)}$ is the exchange-induction energy, $\delta E_{\text {int, }}^{\mathrm{HF}}$ is all the third- and higher-order induction and exchange-induction terms, $E_{\mathrm{intr}, \mathrm{r}}^{\text {(corr) }}$ is the correlated portion of the interaction energy, $E_{\text {int }}($ SAPT2) is the SAPT interaction energy.

表 6 各种能量对总吸引能的贡献比较

Table 6 Comparison of the contribution of the energies to the total attractive energies

\begin{tabular}{ccccc}
\hline Complex & $E_{\text {els }} /\left(\mathrm{kJ} \cdot \mathrm{mol}^{-1}\right)$ & $E_{\text {exch }} /\left(\mathrm{kJ} \cdot \mathrm{mol}^{-1}\right)$ & $E_{\text {ind }} /\left(\mathrm{kJ} \cdot \mathrm{mol}^{-1}\right)$ & $E_{\text {dis }} /\left(\mathrm{kJ} \cdot \mathrm{mol}^{-1}\right)$ \\
\hline $\mathrm{SiH}_{4} \cdots \mathrm{ClF}$ & -14.636 & 58.312 & -35.334 & -14.234 \\
& $(22.8 \%)$ & & $(55.0 \%)$ & $(22.2 \%)$ \\
$\mathrm{SiH}_{4} \cdots \mathrm{BrF}$ & -25.359 & 129.311 & -94.429 & -19.481 \\
& $(18.2 \%)$ & & $(67.8 \%)$ & $(14.0 \%)$ \\
$\mathrm{SiH}_{4} \cdots \mathrm{IF}$ & -28.104 & 162.327 & -125.721 & -20.376 \\
& $(16.1 \%)$ & & $(72.2 \%)$ & $(11.7 \%)$ \\
$\mathrm{SiH}_{4} \cdots \mathrm{ICl}$ & -15.606 & \multirow{2}{*}{80.981} & -56.718 & -15.376 \\
& $(17.8 \%)$ & & $(64.7 \%)$ & $(17.5 \%)$ \\
$\mathrm{SiH}_{4} \cdots \mathrm{BrCl}$ & -11.339 & 51.526 & -32.188 & -13.247 \\
& $(20.0 \%)$ & & $(56.7 \%)$ & $(23.3 \%)$ \\
$\mathrm{HCl} \cdots \mathrm{HCl}$ & -10.581 & 20.292 & -8.121 & -8.293 \\
& $(39.2 \%)$ & & $(30.1 \%)$ & $(30.7 \%)$ \\
\hline
\end{tabular}

$E_{\text {elst }}$ is the electrostatic component of the binding energy; $E_{\text {exch }}$ is the exchange component of the binding energy, $E_{\text {ind }}$ is the induction contribution to the binding energy, $E_{\text {disp }}$ is the dispersion component of the binding energy; the contribution of the energy to the total attractive energy are given in the parentheses.

表现为排斥能.

为了比较反转氢键与常规氢键中各种能量的贡 献情况, 表 5 和表 6 中也列出了同样计算条件得到 的 $\mathrm{HCl}$ 二聚体的能量分解情况. $\mathrm{SiH}_{4} \cdots \mathrm{ClF}$ 体系与 $\mathrm{HCl}$ 二聚体都是 $\mathrm{H}$ 原子与 $\mathrm{Cl}$ 原子作用, 但从表 6 中可看出在 $\mathrm{HCl}$ 二聚体中静电能对总吸引能的贡 献(39.2\%) 占优势, 而 $\mathrm{SiH}_{4} \cdots \mathrm{ClF}$ 体系中诱导能对总 吸引能的贡献(55.0\%)占优势.

\section{3 结 论}

(1) 从 $\mathrm{NBO}$ 原子电荷迁移可以看出, $\mathrm{SiH}_{4}$ 作为 电子给体, 电子迁移的方向与常规氢键相反, 符合
Rozas 等对反转氢键的定义. $\mathrm{NBO}$ 净电荷迁移数从 0.0080 到 0.0246 a.u., 对 $\mathrm{SiH}_{4} \cdots \mathrm{XF}(\mathrm{X}=\mathrm{Cl} 、 \mathrm{Br} 、 \mathrm{I})$ 体系, 随着电荷迁移数的增大, 作用能也增大.

(2) 根据 Bader 的 AIM 理论, 形成常规氢键的条 件是临界点的 $\rho_{\mathrm{c}}$ 在0.002-0.04 a.u.之间, 相应的 $\nabla^{2} \rho_{\mathrm{c}}$ 在 0.024-0.139 a.u.之间. 对所探讨的 $\mathrm{SiH}_{4}$ 与 $\mathrm{AB}$ 型 卤素互化物形成的复合物, $\rho_{\mathrm{c}}$ 从 0.013 到 0.022 a.u., $\nabla^{2} \rho_{\text {c }}$ 从 0.034 到 0.068 a.u., 这说明所研究的 $\mathrm{SiH}_{4}$ 与 $\mathrm{AB}$ 型卤素互化物形成的反转氢键在强度上相当于 常规氢键.

(3) 作用能分解的数据表明, 诱导能对总吸引能 的贡献在 $55.0 \%$ 到 $72.2 \%$ 之间, 是最主要的贡献部 分(常规氢键中静电能对总吸引能的贡献占优势), 在 $\mathrm{SiH}_{4} \cdots \mathrm{IF}$ 体系中, 诱导能对总吸引能的贡献最高, 达到 $72.2 \%$. 在所有体系中, 静电能和色散能对总吸 引能的贡献都小于 $25.0 \%$.

(4) 形成复合物后, 与卤素互化物直接作用的 $\mathrm{Si}-\mathrm{H}$ 键的伸缩振动频率都发生了红移, 没有蓝移 的情况.

\section{References}

1 Jeffrey, G. A. An introduction to hydrogen bonding. Oxford: Oxford University Press, 1997

2 Jeffrey, G. A.; Saenger, W. Hydrogen bonding in biological structures. Berlin: Springer-Verlag, 1991

3 Desiraju, G. R. Crystal engineering. New York: Elsevier, 1989

4 Curtiss, L. A.; Blander, M. Chem. Rev., 1988, 88: 827

5 Custelcean, R.; Jackson, J. E. Chem. Rev., 2001, 101: 1963

6 Klooster, W. T.; Koetzle, T. F.; Crabtree, R. H. J. Am. Chem. Soc., 1999, 121: 6337 
7 Desiraju, G. R.; Steiner, T. The weak hydrogen bond in structural chemistry and biology. New York: Oxford University Press, 1999

8 Grabowski, S. J.; Sokalski, W. A.; Leszczynski, J. J. Phys. Chem. A, 2004, 108: 5823

9 Zhu, W. L.; Puah, C. M.; Tan, X. J.; Jiang, H. L.; Chen, K. X. J. Phys. Chem. A, 2001, 105: 426

10 Liu, H.; Chen, Y. Q. Acta Phys. -Chim. Sin., 2007, 23: 1974 [刘 红, 陈燕芹. 物理化学学报, 2007, 23: 1974]

11 Liu, H.; Chen, Y. Q.; Wang, Y. B. Acta Chim. Sin., 2008, 66: 301 [刘 红, 陈燕芹, 王一波. 化学学报, 2008, 66: 301]

12 Kulkarni, S. A.; Srivastava, A. K. J. Phys. Chem. A, 1999, 103, 2836

13 Liu, H.; Chen, Y. Q.; Yang, Y. Q.; Chen, J. Computers and Applied Chemistry, 2008, 25: 1405 [刘 红, 陈燕芹, 杨玉琼, 陈 健. 计算机与应用化学, 2008, 25: 1405]

14 Rozas, I.; Alkorta, I.; Elguero, J. J. Phys. Chem. A, 1997, 101: 4236

15 Li, W. K.; Zhou, G. D.; Mak, T. C. Advanced structural inorganic chemistry. New York: Oxford University Press, 2008: 415

16 Blanco, F.; Solimannejad, M.; Alkorta, I. Theoretical Chemistry Accounts, 2008, 121: 3
17 Alkorta, I.; Rozas, I.; Elguero, J. J. Phys. Chem. A, 2001, 105: 743

18 Alkorta, I.; Rozas, I.; Elguero, J. International Journal of Quantum Chemistry, 2002, 86: 122

19 Frisch, M. J.; Trucks, G. W.; Schlegel, H. B.; et al. Gaussian 98. Revision A.01. Pittsburgh, PA: Gaussian Inc., 1998

20 Biegler-Konig, F. AIM2000. Bielefeld, Germany: University of Applied Sciences, 2000

21 Szalweicz, K.; Bukowski, R.; Cencek, W.; et al. SAPT2002: an $a b$ initio program for many-body symmetry-adapted perturbation theory calculations of intermolecular interaction energies. University of Delaware and University of Warsaw. http://www. physics.udel.edu/ szalewic/SAPT/SAPT.html

22 Bader, R. F. W. Atoms in molecules: a quantum theory. Oxford: Oxford Unversity Press, 1990

23 Reed, A. E.; Curtiss, L. A.; Weinhold, F. Chem. Rev., 1988, 88: 899

24 Wu, Y.; Feng, L.; Zhang, X. D. Acta Phys. -Chim. Sin., 2008, 24 653 [吴 阳, 冯 璐, 张向东. 物理化学学报, 2008, 24: 653]

25 Liang, X.; Wang, Y. B. Acta Chim. Sin., 2008, 66: 1385 [梁 雪, 王一波. 化学学报, 2008, 66: 1385] 